\section{Does the bacterial media culture chemistry affect the stability of nanoparticles in nanotoxicity assays?}

\author{
M. Millour, K. Doiron, K. Lemarchand, \\ J.-P. Gagné \\ Institut des sciences de la mer de \\ Rimouski, Université du Québec à \\ Rimouski, Rimouski, Québec, Canada
}

\section{Introduction}

New classes of silver nanoparticles (AgNPs) with antimicrobial activities are under rapid development for incorporation into consumer products. Important applications are in food packaging materials, odor-resistant textiles, paints, cosmetics, medical devices, water disinfectants, etc. ${ }^{1}$ This increasing use of AgNPs raised the issue of the release of nanosilver from a variety of sources, under different forms, into aquatic environments. Recent studies have confirmed the release of AgNPs from socks and nanotextiles during washing ${ }^{2,3}$ or from surfaces coated by nanosilver paints during rain-water runoff events. ${ }^{4}$ The environmental effects of the AgNPs discharged into aquatic environments are largely unknown. ${ }^{1,5}$ However, laboratory ecotoxicological studies on fishes, crustaceans, algae and bacteria have shown toxic effects on test organisms exposed to AgNPs. ${ }^{1,5-7}$ Due to the antimicrobial properties of AgNPs, it is important to understand which factors control their toxicity toward environmental microorganisms since they play important roles in major biogeochemical cycles, in the production of natural organic matter and in the recycling of nutrients. Bacterial sensitivity to silver nanoparticles and ionic silver have been demonstrated on reference bacterial strains of the genera Bacillus, Escherichia, Pseudomonas and Vibrio ${ }^{7-10}$ as well as on bacterial communities. ${ }^{6,11,12}$ Today, there is no consensus on the mechanisms of antimicrobial action of AgNPs. Proposed processes ${ }^{1,8-10,13}$ include: i) free silver ion dissolution from AgNPs followed by cellular uptake and disturbance of intracellular reactions; ii) cellular uptake of AgNPs followed by reactive oxygen species (ROS) generation and release of silver ions impacting DNA replication and ATP production; and iii) direct interaction of AgNPs with cell surface altering protein function and membrane permeability. These processes were demonstrated to be dependent on nanoparticle's size and specific properties (shape, chemical composition, surface charge, coating, etc.). Moreover, the chemical composition of exposure media $(\mathrm{pH}$, ionic strength, ionic composition, quality and quantity of natural organic matter) is also a relevant factor that would affect the toxicity of AgNPs. The solution chemistry could play an important role in the aggregation and in the fate of AgNPs. If aggregation occurs, the size of AgNPs in solution will change, modifying the sedimentation and the ability of AgNPs to interact with proteins at the cellular surface or to cross-cellular membranes. Thus, aggregation can modulate the toxicity of nanoparticles. Considering that the size of AgNPs can be a significant factor controlling the toxicity of silver nanoparticles, it is important to understand how the exposition media (e.g., culture broths) modify the size of the nanoparticles used in toxicity assays. The purpose of this study is to assess the stability of AgNPs suspensions with time in different exposure solutions characteristic of natural waters or of media currently used in microbial ecotoxicology studies. We aim to show the importance to control the aging of AgNPs colloidal suspensions during toxicity assays.

\section{Materials and Methods}

All experiments were conducted with citrate coated silver nanoparticles obtained by the reduction of $\mathrm{AgNO}_{3}$ with sodium citrate. Suspension of AgNPs was purified to remove excess of $\mathrm{AgNO}_{3}$ and citrate and to concentrate AgNPs. AgNPs synthetized had a hydrodynamic diameter of $9 \pm 2 \mathrm{~nm}$. Salts used in this study were $\mathrm{NaCl}, \mathrm{CaCl}_{2}, \mathrm{MgCl}_{2}$. Artificial seawater (ASW) was made by mixing $400 \mathrm{mM}$ of $\mathrm{NaCl}, 41.4 \mathrm{mM}$ of $\mathrm{MgSO}_{4} .7 \mathrm{H}_{2} \mathrm{O}$ and $1.8 \mathrm{mM}$ of $\mathrm{NaHCO}_{3}$. Salt solutions and ASW were prepared and regulated at pH 7 and at 25\%. This salinity is characteristic of estuarine environments. These solutions were filtered on 0.2$\mu \mathrm{m}$ pore-sized polycarbonate membrane. Culture media tested were Guillard's F/2, generally used for marine microalgae culture, and Luria Broth (LB), commonly used for bacterial growth. F/2 media was prepared by diluting trace element (nitrate, phosphate, metal and vitamins) in ASW. LB media was composed of $10 \mathrm{~g}$ of tryptone, $5 \mathrm{~g}$ of yeast extract and $10 \mathrm{~g}$ of $\mathrm{NaCl}$ for $1 \mathrm{~L}$ of nanopure water at $\mathrm{pH}$ 7.2. The evolution of hydrodynamic diameters of the AgNPs suspensions at concentrations of $93 \mu \mathrm{gAg} . \mathrm{L}^{-1}$ was measured by time resolved dynamic light scattering (TR-DLS) using Zetasizer Nano ZS (Malvern Instruments Ltd., Malvern, Worcestershire, UK) instrument during $17 \mathrm{~h}$ at $25^{\circ} \mathrm{C}$. Parameters used for the TR-DLS was one measure each 10 min with an angle of $173^{\circ}$. One measure was obtained with 10 scans of $15 \mathrm{~s}$ each. The concentration used (93 $\left.\mu \mathrm{gAg} . \mathrm{L}^{-1}\right)$ in the experiments was in the lower range of concentrations used to test the toxicity of AgNPs to microorganisms.
Correspondence: Jean-Pierre Gagné, Institut des sciences de la mer de Rimouski, Université du Québec à Rimouski, 310 Allée des Ursulines, C.P. 3300, Rimouski, Québec, Canada, G5L 3A1. Tel: 1.418.723.1986 ext. 1870 - Fax: 1.418.724.1842. E-mail: jean-pierre_gagne@uqar.ca

Key words: Citrate-coated silver nanoparticles; aggregation; salt effects; culture media effects.

Acknowledgments: this work was supported by Strategic Project Grants of the National Sciences Engineering Research Council of Canada (Émilien Pelletier and Jean-Pierre Gagné), Discovery Project Grants of the National Sciences Engineering Research Council of Canada (JeanPierre Gagné) and Québec-Océan for its financial support.

Conference presentation: part of this paper was presented at ECOBIM meeting, 2015 May, Québec City, Québec, Canada.

This work is licensed under a Creative Commons Attribution NonCommercial 3.0 License (CC BYNC 3.0).

(C) Copyright M. Millour et al., 2015

Licensee PAGEPress, Italy

Journal of Xenobiotics 2015; 5:5772

doi:10.4081/xeno.2015.5772

\section{Results and Discussion}

Figure 1 shows the evolution in hydrodynamic diameter (Dh) for AgNPs over $17 \mathrm{~h}$ in nanopure water, $\mathrm{NaCl}, \mathrm{CaCl}_{2}$ or $\mathrm{MgCl}_{2}$ solution. In nanopure water, the Dh of AgNPs remained stable at $9 \pm 2 \mathrm{~nm}$ during $17 \mathrm{~h}$ (Figure 1A). In presence of $\mathrm{NaCl}$ at $25 \%$, the Dh of AgNPs (9 $\mathrm{nm}$ ) evolved rapidly (Figure 1B) to reach $550 \pm 280 \mathrm{~nm}$ after $100 \mathrm{~min}$. When $\mathrm{CaCl}_{2}$ was added to nanopure water at $25 \%$, the Dh of AgNPs increased rapidly during the first 50 min (Figure 1C) suggesting that the rate of AgNPs aggregation is modulated by the nature of cations in solution. After $50 \mathrm{~min}$, the Dh did not evolved anymore and stabilized at $424 \pm 69$ $\mathrm{nm}$. In presence of $\mathrm{MgCl}_{2}$ added to nanopure water at 25\%0, the behavior of AgNPs was the same as in the $\mathrm{CaCl}_{2}$ solution (Figure 1D). After the rapid increase (50 min), the $\mathrm{Dh}$ remained at $548 \pm 105 \mathrm{~nm}$. The rapid, but different, increases of Dh observed in $\mathrm{NaCl}, \mathrm{CaCl}_{2}$ and $\mathrm{MgCl}_{2}$ solutions reflected the aggregation of AgNPs in presence of various cations and showed that divalent cations were more effective in accelerating aggregation than monovalent cations.

Figure 2 presents the time evolution of AgNPs hydrodynamic diameter in ASW, F/2 and LB media. In ASW, from 0 min to 200 min, the Dh increased rapidly from $9 \mathrm{~nm}$ to near $400 \mathrm{~nm}$ 
as shown in Figure 2A. After 200 min the Dh reached $422 \pm 255 \mathrm{~nm}$. In F/2 medium (Figure 2B), the behavior of AgNPs was similar to that observed in ASW. A rapid increase in Dh was observed during the first 200 min afterward the Dh remained at $635 \pm 253 \mathrm{~nm}$. In LB broth, the Dh of AgNPs increased rapidly during the first $250 \mathrm{~min}$. Later on, the diameter stabilized around $511 \pm 365 \mathrm{~nm}$. In complex solutions (Figure 2) slower changes in aggregation rates were observed compared to those observed in simple solutions (Figure 1) during the first $100 \mathrm{~min}$. This involves that for a complex aqueous solution, the rate of aggregation is modified by the presence of cations but also by other components, such as organic matter (vitamins in F/2 medium and tryptone and yeast extract in LB medium) present in the culture media.

Stability or aggregation of AgNPs could be rationalized, in first approximation, by the Derjarguin, Landau, Verwey and Overbeek (DLVO) theory. ${ }^{14}$ Stability is controlled by attractive and repulsive interactions. Attractive interactions are van der Waals forces and repulsive interactions are governed by the electrical double layer. ${ }^{14}$ When repulsive interactions are greater than attractive interactions, the conditions are unfavorable for aggregation, but when repulsive interactions are equal or lower than attractive forces, the aggregation is promoted. The transition between these two steps is characterized by the critical coagulation concentration (CCC). For AgNPs coated with citrate, the CCC was generally close to $50 \mathrm{mM}$ for $\mathrm{NaCl}$ and near 2 $\mathrm{mM}$ for divalent cations ${ }^{15}$ present in $\mathrm{CaCl}_{2}$ or $\mathrm{MgCl}_{2}$ solution. In this study, the $\mathrm{pH}$ solution was near 7 and the salt concentrations were greater than the CCC, thus aggregation occurs. In nanopure water, the citrate moieties of AgNPs were negatively charged. This promotes the electrostatic repulsions between AgNPs, thus avoiding the aggregation of AgNPs. ${ }^{14,16}$ Moreover, the presence of salts has increased the ionic strength of the solution decreasing the thickness of the electrical double layer around the silver nanoparticles. This has enhanced the close encounter of AgNPs and therefore the aggregation. However, the presence of salts also affected the charges at the surface of AgNPs. Citrate moieties were negatively charged and can interact with cations. Interactions between citrate and cations decreased the negative charges of AgNPs and lowered the electrostatic repulsion between particles. $^{14,16}$ These phenomena enhanced the aggregation of $\mathrm{AgNPs}^{17}$ and can explain the behavior of AgNPs in nanopure water and salt solutions. Another process that could contribute to the aggregation was the presence of multivalent cations. Those cations could form a bridge between two adjacent AgNPs facilitating the aggregation of nanoparticles ${ }^{16}$. The dif- ference observed in the rapid increase in Dh of AgNPs in presence of $\mathrm{NaCl}$ and $\mathrm{CaCl}_{2}$ or $\mathrm{MgCl}_{2}$ could be explained by this process.

In complex media such as ASW, F/2 or LB, the aggregation rate was lower than in salt solutions. Jin et al. ${ }^{8}$ have discussed the effects of synthetic freshwater composition on aggregation and dissolution of AgNPs. When freshwater contained the same salts as those in ASW, the aggregation of AgNPs was modified.
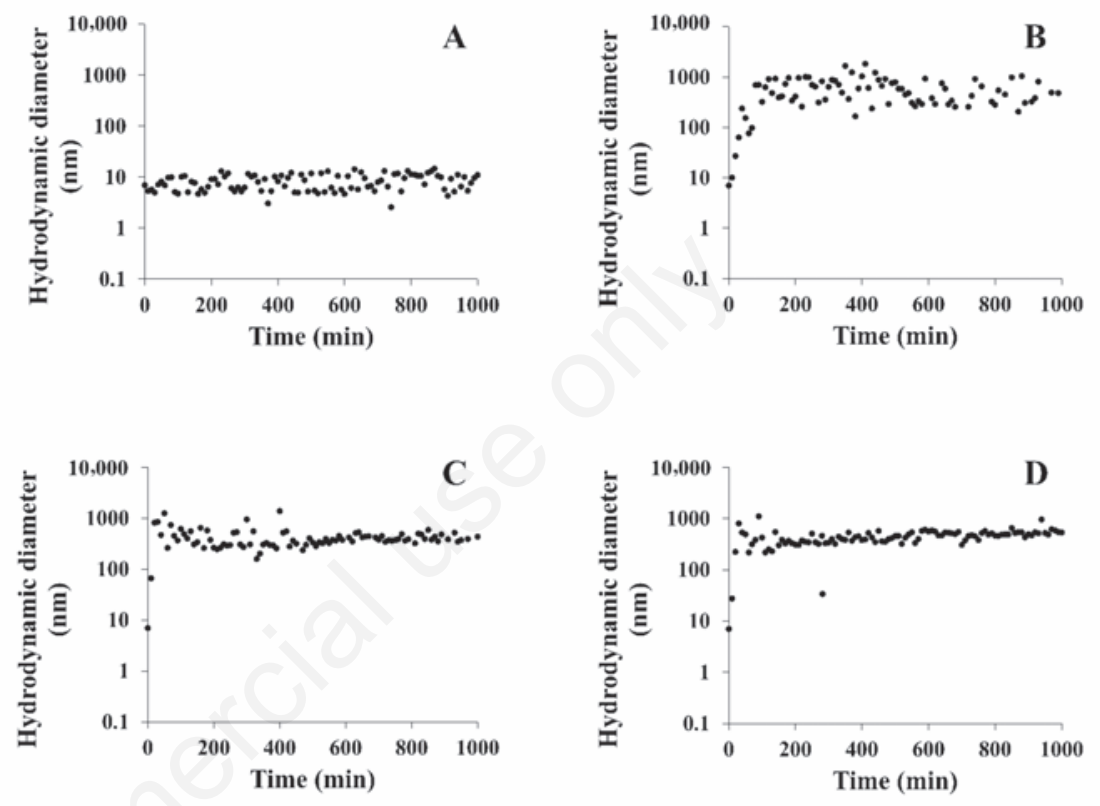

Figure 1. Aggregation kinetics of silver nanoparticles at $93 \mu \mathrm{gAg} . \mathrm{L}^{-1}$ in: A) nanopure water; B) $\mathrm{NaCl}$ at $25 \%$; C) $\mathrm{CaCl}_{2}$ at $25 \%$; and D) $\mathrm{MgCl}_{2}$ at $25 \%$.
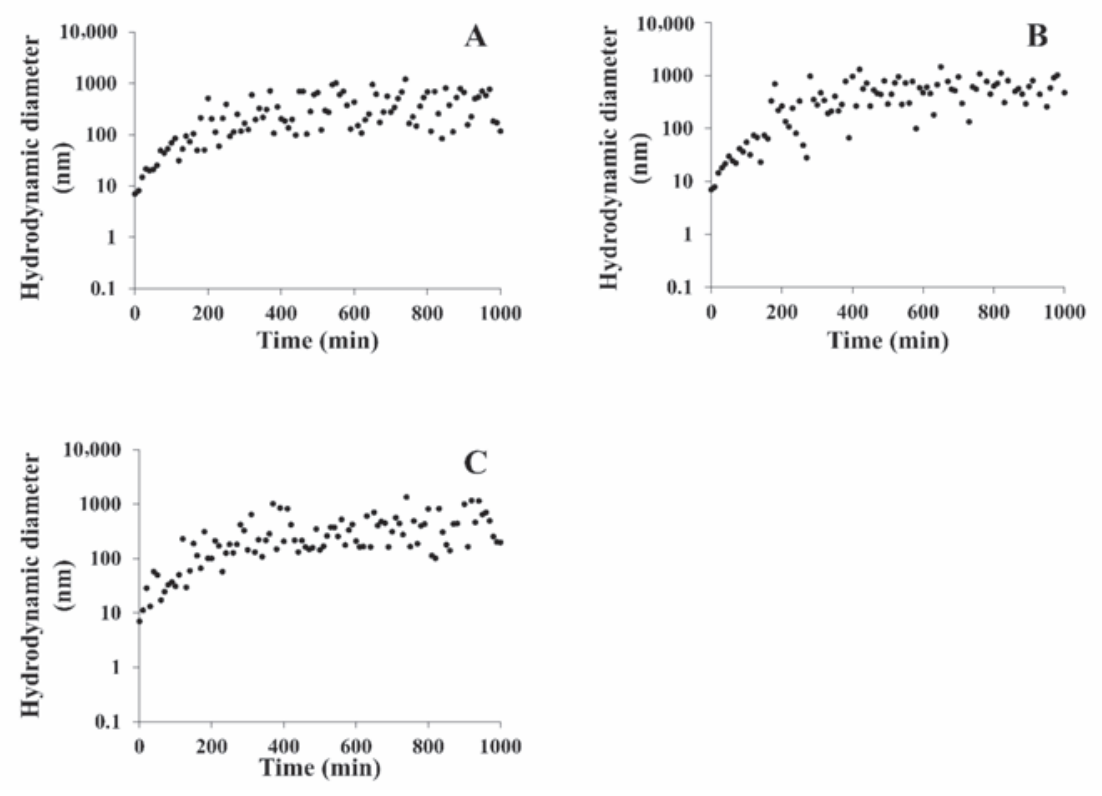

Figure 2. Aggregation kinetics of silver nanoparticles at $93 \mu \mathrm{gAg} . \mathrm{L}^{-1}$ in: A) artificial seawater; B) F/2 media; and C) LB media.

[Journal of Xenobiotics 2015; 5:5772]
This modification was caused by the reaction of $\mathrm{Ag}^{+}$, released from $\mathrm{AgNPs}$, with anions like $\mathrm{Cl}^{-}$or $\mathrm{HCO}_{3}^{-}$. The salts formed during the reaction were insoluble and could interact with the surface of AgNPs to create a new coating. ${ }^{18}$ With this new coating, the toxicity of nanoparticles could be different than the toxicity of the ative nanoparticles.

F/2 medium, the trace element concentration could be too low to affect AgNPs aggrega- 
tion. The behavior of AgNPs in F/2 medium was controlled by the most important cation, $\mathrm{Na}^{+}$, in this culture broth. The anions, such as $\mathrm{Cl}^{-}$and $\mathrm{HCO}_{3}^{-}$, can precipitate with silver ions around the AgNPs as described for ASW. The LB medium has a concentration in $\mathrm{NaCl}$ near $171 \mathrm{mM}$, higher than the CCC. However, the presence of organic matter ( $\mathrm{OM})$, like tryptone and yeast extract, could affect the aggregation of AgNPs by increasing the CCC. The addition of $\mathrm{OM}$ could create a new coating on AgNPs. ${ }^{15}$ This new coating would modify the interactions between AgNPs, OM and ions in solution thereby producing a new equilibrium characterized by a decrease of AgNPs aggregation rate. This hypothesis could explain the difference in the time evolution of AgNPs size observed in this study.

As shown in Figures 1 and 2, aggregation of nanoparticles is a common phenomenon in complex aqueous environments. The rapid changes in size observed during the first hours of our experiments are related to the charge of the cations, the presence of $0 \mathrm{M}$ in the culture media, the nature of nanoparticles and the contact time between nanoparticles and the surrounding solution. A steady state in the size of the aggregates has been observed after different time periods, from $50 \mathrm{~min}$ in solution of divalent cations to about 200-250 min in culture broth F/2 or LB. In the steady state, for ASW, LB and F/2 respectively, the Dh of the aggregates increased by 46,57 and 70 fold compared to the nominal Dh of AgNPs. This can have a strong incidence on the toxicity. For instance, the increase in $\mathrm{Dh}$ in $\mathrm{F} / 2$ medium decreased the number of nanoparticles by about 10,000 between the beginning and the end of the experiment. Such variations in particle numbers, in the size and in the time period where aggregation occur can lead to misrepresentative results or interpretations if toxicological assays are discussed without an indepth description of conditions used in experiments. The time between the addition of nanoparticles to culture media and the duration of the toxicological assay should be clearly mentioned in the materials and methods of any nanotoxicological study.

\section{Conclusions}

Aggregation of AgNPs could have effects on microalgae and bacterial growth and as a consequence modulates the AgNPs toxicity. The growth of these organisms was characterized by four steps: lag phase, exponential phase, stationary phase and decline or death phase. Aggregation of AgNPs could extend the lag phase or decrease growth rate of cultured microorganisms since they need to adapt to the evolution of AgNPs size and particle numbers in solution. During the rapid increase of Dh of AgNPs, the culture media are in constant evolution. This could increase the lag phase as observed in some studies. ${ }^{6,7}$ During aggregation, the toxicity mechanism could change. The small aggregates of AgNPs could penetrate in the cell and produce ROS inside the cell. When the aggregates become larger they cannot penetrate the cell but can interact with their surface and alter membrane permeability and protein functions. The decrease of particle concentration can also modify the response of organisms exposed to AgNPs. Depending of the aggregation state of the AgNPs suspension, toxicity of AgNPs could be different. This study demonstrates that characterizing the behavior of AgNPs in the toxicity assay media is essential to better understand the real effects of AgNPs toward microorganisms.

\section{References}

1. SCENIHR (Scientific Committee on Emerging and Newly Identified Health Risks). Nanosilver: safety, health and environmental effects and role in antimicrobial resistance. Luxembourg: SCENIHR; 2014. Available from: http://ec.europa. eu/health/ scientific_committees/emerging/docs/scenihr_0_039.pdf

2. Benn TM, Westerhoff P. Nanoparticle silver released into water from commercially available sock fabrics. Environ Sci Technol 2008;42:4133-9.

3. Geranio L, Heuberger M, Nowack B. The behavior of silver nanotextiles during washing. Environ Sci Technol 2009;43: 8113-18.

4. Kaegi R, Sinnet B, Zuleeg S, Hagendorfer H, Mueller E, Vonbank R, et al. Release of silver nanoparticles from outdoor facades. Environ Pollut 2010;158:2900-5.

5. Stone V. ENRHES: Engineered nanoparticles: review of health and environmental safety; Edinburg, 2009. Available from: http://cordis.europa.eu/result/rcn/45841_e n.html

6. Doiron K, Pelletier E, Lemarchand K. Impact of polymer-coated silver nanoparticles on marine microbial communities: a microcosm study. Aquat Toxicol 2012;124125:22-7.

7. Doiron K, Millour M, Gagné J-P, Lemarchand K. Combined effects of silver nanoparticles and humic and fulvic acids on Vibrio splendidus growth. J Xenobiotics
2014;4:4893.

8. Jin X, Li M, Wang J, Marambio-Jones C, Peng F, Huang $X$, et al. High-throughput screening of silver nanoparticle stability and bacterial inactivation in aquatic media: Influence of specific ions. Environ Sci Technol 2010;44:7321-8.

9. Chambers BA, Afrooz ARMN, Bae S, Aich N, Katz L, Saleh NB, et al. Effects of chloride and ionic strength on physical morphology, dissolution, and bacterial toxicity of silver nanoparticles. Environ Sci Technol 2014;48:761-9.

10. Marambio-Jones C, Hoek EMV. A review of the antibacterial effects of silver nanomaterials and potential implications for human health and the environment. J Nanoparticle Res 2010;12:1531-51.

11. Das P, Xenopoulos MA, Williams CJ, Hoque ME, Metcalfe CD. Effects of silver nanoparticles on bacterial activity in natural waters. Environ Toxicol Chem 2012;31: 122-30.

12. Das P, Williams CJ, Fulthorpe RR, Hoque ME, Metcalfe CD, Xenopoulos MA. Changes in bacterial community structure after exposure to silver nanoparticles in natural waters. Environ Sci Technol 2012; 46:9120-8.

13. Lapresta-Fernández A, Fernández A, Blasco J. Nanoecotoxicity effects of engineered silver and gold nanoparticles in aquatic organisms. Trends Anal Chem 2012;32:40-59.

14. Petosa AR, Jaisi DP, Quevedo IR, Elimelech $\mathrm{M}$, Tufenkji N. Aggregation and deposition of engineered nanomaterials in aquatic environments: role of physicochemical interactions. Environ Sci Technol 2010;44: 6532-49.

15. Huynh KA, Chen KL. Aggregation kinetics of citrate and polyvinylpyrrolidone coated silver nanoparticles in monovalent and divalent electrolyte solutions. Environ Sci Technol 2011;45:5564-71.

16. El Badawy AM, Luxton TP, Silva RG, Scheckel KG, Suidan MT, Tolaymat TM. Impact of environmental conditions $(\mathrm{pH}$, ionic strength, and electrolyte type) on the surface charge and aggregation of silver nanoparticles suspensions. Environ Sci Technol 2010;44:1260-6.

17. Zhang Y, Chen Y, Westerhoff P, Crittenden J. Impact of natural organic matter and divalent cations on the stability of aqueous nanoparticles. Water Res 2009;43:4249-57.

18. Levard C, Hotze EM, Lowry GV, Brown GE. Environmental transformations of silver nanoparticles: impact on stability and toxicity. Environ Sci Technol 2012;46:6900-14. 